\title{
SKEUOMORPHS AND REMEDIATIONS: WORKING ENVIRONMENTS FOR CONSUMER VIRTUAL REALITY PLATFORMS
}

\begin{abstract}
Acknowledging the latest renaissance of spatial and immersive media technologies and content (VR/AR), the article reconceptualizes their status as software media and cultural objects. Specifically, drawing on work of i.a. Lev Manovich, Alexander Galloway and Oliver Grau, the author argues that due to a wide range of real-time sensory cues that a VR experience delivers, it should be considered as a new type of software media interface, rather than a multisensory projection. The argument is based on case study research on commercially available software applications that strive for converting computer-generated spatial immersive environments into working environments. Consumer-oriented software was selected as the object of study due to its exposure. Consequently, its popularity based i.a. on low entry barrier, leads to a greater social and cultural impact than in-house, corporate VR software or immersive new media arts projects do.

The study focuses particularly on design principles implemented to create a virtual working environment and on interaction methods used as HCI paradigms for managing the data and navigating the environment. In order to achieve a certain level of familiarity and support for legacy data and media, the aesthetics of VR working environments is based i.a. on spatial remediations of established GUI elements and visual skeuomorphism. The analysis shows that VR as a relatively new medium, particularly in non-gaming applications, faces challenges in the area of pre-VR content representation, on one hand, and in taking advantages of the affordances offered by a spatial environment (particularly at the interaction and level), from the other. Theoretically, the study follows software and platform studies approaches, informed by critical theory and media studies perspectives.
\end{abstract}

Keywords: virtual reality, interface, interaction, software studies, remediation

\section{Visual interfaces in personal computing}

Decades after it was introduced by Xerox PARC, Apple and Microsoft, the Graphical User Interface (GUI) based on Windows, Icons, Mouse, Pointer (WIMP) paradigm has dominated general purpose computing and human-computer interaction. 
Text and visual metaphor-based interface controlled by mouse and keyboard has only recently begun to be challenged by new multi-touch interaction and new interface devices (e.g. voice and touch interfaces). ${ }^{1}$ Different GUIs are today applied in various desktop, mobile or wearable devices, and despite major differences in their form factor and use cases, all of these devices present visual content to users using a framed, far-eye, flat display surface. However, the latest revival of immersive media technologies, like Virtual or Augmented Reality (VR/AR) that use near-eye displays (e.g. Head Mounted Displays, semi-translucent smart glasses), which completely or partially block users' visual cues on physical world, pose a challenge to established conventions of GUI design and content display. VR in particular, considered as a medium offering interactive and immersive computing experience that can become a working environment, requires a new approach to human-computer interface design. This approach should take into account the affordances and limitations coming from spatial and real-time content representation and interaction that VR is based on.

The paper aims to reconceptualize spatial and immersive media technologiesparticularly virtual reality (VR). I argue that due to a wide range of real-time sensory cues that a VR experience delivers and its ability to present information and content spatially, it should be considered as a new type of software media interface, rather than a multisensory projection. The argument is based on a case study research of consumer-oriented applications and interfaces for visualizing and interacting with virtual reality spatial environments designed as working or productivity environments. This particular type of VR software was chosen based on its social and cultural exposure. These commercially available products may have a more significant and long-lasting influence on cultural and social impact of VR software, than in-house, corporate VR software or immersive media arts projects.

\section{The conditions behind the revival of immersive media- standardization and protocolization}

Before focusing on the case study material, we should briefly discuss the key terms referenced in the paper and then proceed to an overview of techno-cultural conditions behind the latest revival of immersive media. Jason Jerald defines virtual reality (VR) as "a computer-generated digital environment that can be experienced and interacted with as if that environment were real" and augmented reality (AR) as "[a technology that] adds cues onto the already existing real world, and ideally the human mind would not be able to distinguish between computer-generated stimuli

K. Akeley, A. van Dam, J.D. Foley, S.K. Feiner, J.F. Hughes, M.McGuire, D. Sklar, Computer Graphics: Principles and Practice, Boston, MA: Addison-Wesley Longman Publishing Co., Inc., 2013, p. 568. 
and the real world". ${ }^{2}$ This paper focuses on VR; however, AR will be referenced a few times as the development process behind these technologies (for instance the tools used to produce 3D models, certain protocols or standards) is to some extent shared by both of them. Clearly, the end product of this creative process and hardware involved, be it an application, a narrative or a game, is radically different. A VR application aims to create a complete virtual environment aiming for immediacy, while an AR application only puts (superimposes) certain elements or information into a real environment experienced by a user, thus following the logic of hypermediacy. ${ }^{3}$

The latest revival of consumer immersive media technologies began in 2014 when Facebook bought Oculus - a small start-up working on HMD VR glasses in $2014 .{ }^{4}$ This triggered a series of events in the IT industry and after 20 years of stagnation, VR has become a mainstream topic again. Obviously, there is a convergence of several technological and cultural factors that lie behind the renaissance of all the immersive media technologies (VR, AR, MR) in the reality-virtuality continuum. ${ }^{5}$ I argue that the revival of interest in consumer-oriented VR has been possible mainly by market availability of relatively inexpensive, well designed and easy to use standardized devices. Standardized devices provide a technological and business structure for VR content creators and software developers, functioning as an easy-accessible channel to deliver their products to millions of consumers. In response to the affordances and limitations of new hardware, software companies started to offer VR support in software suites for creating 3D real-time experiences. For instance, Unity 3D and Unreal Engines, 3D content creation suites, that are used both by major studios and by indie developers, introduced official VR support (OpenVR - HTC / SteamVR; Google VR SDK - Daydream, Cardboard; Oculus - Rift, Gear VR, Windows Mixed Reality) as early as in $2012^{6}$ and in $2015 .^{7}$ Consequently, a fairly straightforward development pipeline for VR content has been established, and the need for designing graphical user interfaces for this content emerged.

The significance of a standardized device for new developments and particularly, for mass-scale adoption of new computational devices and products has been emphasized i.a. by Matthew Fuller. David M. Berry also writes that "digital technologies

J. Jerald, The VR Book: Human-Centered Design for Virtual Reality, New York: ACM Books, 2016, pp. 9, 29.

3 J.D. Bolter, R. Grusin, Remediation: Understanding New Media, Cambridge, MA: MIT Press, 2003, pp. 272-273.

4 A. Heath, "Mark Zuckerberg reveals that Facebook paid more than we thought for Oculus VR", Business Insider 2017, https://www.businessinsider.com/facebook-actually-paid-3-billion-for-oculus-vr-2017-1.

5 P. Milgram et al., "Augmented Reality: A Class of Displays on the Reality-Virtuality Continuum", Proceedings of Telemanipulator and Telepresence Technologies 1994, pp. 2351-2334.

6 T. Andrade, "Creating a Basic VR Demo Using Unreal", Virtual Reality Pop 2016, https://virtualrealitypop.com/start-a-vr-demo-using-the-unreal-engine-63d31eeaf784.

7 B.P. Rubin, "Unity Dives into the Rift with Virtual Reality Tools for Games", ReadWrite 2015, https:// readwrite.com/2015/06/10/unity-adds-vr-support-to-game-engine/. 
as such are based on a constellation of standards, canonical ways of passing around discrete information and data." "According to Fuller, standard objects have become crucial to the generation of media and communications networks and the organizations that handle them. Standard objects should be understood as "isolated systems, separate entities within the universe [...] There are truths respecting this system which require reference only to the remainder of things by way of a uniform systematic scheme of relationships. Key examples of standard objects are the freight container and digital packet switching, compression algorithms, etc." Another phenomenon that is correlated with standardization in the field of computer and software engineering is the notion of protocol. Protocols can be characterized as technical standards that govern the implementation of specific technologies. ${ }^{10}$ The TCP/IP model of Internet layering or e-mail IMAP protocol are one of the most well-known implementation of protocols that allow to exchange data between various interconnected devices and software environments. However, despite the seemingly openness of the protocol, at the heart of it lies a contradiction-in fact protocols impose strict regulations on parties that are interested in using them as an underlying structure. Galloway claims that "[protocol] has to standardize in order to liberate." 11 Protocols use "politically reactionary tactic that enables radical openness"12 only through outlining clear requirements for a designed form for occurrences that are taking place within them. Linking this observation with the emerging VR industry, it seems that the main difference between today's developments and achievements from the early $90 \mathrm{~s}$ is the fact that nowadays it is striving towards standardization and protocolization-like the OpenXR initiative led by Khronos Group. ${ }^{13}$ By 2019 the standard is going to offer a framework for VR/AR applications development and delivery across a wide variety of hardware and software immersive media platforms. ${ }^{14}$ Even though, as of 2018 there are multiple (competing) hardware and software VR solutions, they are all based on fairly similar "building blocks" backed by certain standardized software and hardware components.

From a consumer's perspective, it seems that the emerging VR industry is trying to replicate the strategy that turned out to be successful in the mobile market, where thanks to standardized software ecosystems offered by Apple and Google, developers have been able to create millions of applications offering extremely diversified use

D.M. Berry, Critical Theory and the Digital, London: Bloomsbury, 2014, p. 10.

9 M. Fuller, Media Ecologies: Materialist Energies in Art and Technoculture, Cambridge, MA: MIT Press, 2007, p. 93.

10 A.R. Galloway, Protocol, or, How Control Exists After Decentralization, Cambridge, MA: MIT Press, 2001, p. 7.

$11 \quad$ Ibid., p. 95.

12 Ibid., p. 145.

13 Khronos Group, “OpenXR Overview”, https://www.khronos.org/openxr.

14 N. Whiting, "Standardizing All the Realities: A Look at OpenXR", GDC 2018, https://www.youtube. $\mathrm{com} /$ watch? $\mathrm{v}=\mathrm{U}-\mathrm{CpA} 5 \mathrm{~d} 9 \mathrm{MjI}$. 
cases. At a hardware level, today's VR industry, draws from the achievements of mobile industry (fairly cheap, miniaturized processing units, sensors and display). As a result, hardware wise, headsets offered by any major vendor-HTC, Oculus, Sony - are relatively similar and thus they offer a comparable immersive experience. The software layer of today's VR products is more diversified. Several key actors are trying to position their software ecosystem as the dominant means for content creation (Unity 3D, Unreal, Vuforia) and distribution/access (SteamVR, VIVEPORT, PlayStation Store). Acknowledging the proprietary strategies introduced by hardware vendors (in particular at the stage of content distribution - dedicated digital store that is promoted by each producer), the general context of producing and distributing immersive media experiences radically differs from the reality of the early $90 \mathrm{~s}$. Back at that time, digital artist Charlotte Davies and her team had to design and rely on custom hardware and software platform in order to produce just a single artworkOsmose..$^{15}$ It is worth noting that the artwork is still considered to be one of the most compelling immersive experiences even created. With all these advancements in hardware, software and in the content development pipeline, the industry's assumption is that VR may finally become not only a niche technology used by military and academic communities, but also a general purpose software platform that would offer real-time highly interactive experiences to players and virtual working environments to users alike. ${ }^{16}$ With that in mind, we could start developing an idea for VR becoming not only a computer platform, but also a new real-time spatial media interface.

\section{VR as an immersive computing interface}

Alexander Galloway claims that interfaces (specifically software interfaces) should be considered more as "processes that effect a result", autonomous zones of activity, than static objects or mere software layers. ${ }^{17}$ Today's GUIs are multi-media representational structures that allow to display and manipulate different data in realtime. They use certain culturally rooted design patterns like icons or windows to provide a structure for navigation and data representation. The role of cultural conventions is crucial in interface design, as technically speaking "one (digital) database can be accessed with different types of media interfaces" 18 and data as such "have no necessary visual form" 19 , therefore the interface is constrained more by non-technical, cultural factors than by software limitations.

15 Ch. Davies, Osmose, http://www.immersence.com/osmose/.

16 See: S. Israel, R. Scoble, The Fourth Transformation: How Augmented Reality \& Artificial Intelligence Will Change Everything, Austin: Patrick Brewster Press, 2016; J. Jerald, op. cit.

17 A.R. Galloway, The Interface Effect, Cambridge, UK: Polity, 2012, pp. VII, 36.

18 L. Manovich, The Language of New Media, Cambridge, MA: MIT Press, 2001, p. 57.

19 A. Galloway, The Interface Effect, p. 82. 
Following Galloway's idea of interfaces as zones of activity, we could consider immersive CGI-based environments, like VR, not as multi-sensory projections, but rather as interactive, real-time interfaces. In fact, the key characteristic behind VR is that it is a real-time (dynamic) and multi-sensual (multi-media) medium, where thanks to a projection of convincing stimuli, a user can feel a sense of presence inside a virtually created space. Bolter and Grusin explicitly say that "the responsive character of the environment, gives VR its sense of presence." ${ }^{\prime 20}$ In contrast to presence which is a subjective, internal psychological state, immersion is an objective degree. The level of immersion, which is in itself a technical characteristic, is determined by the affordances and capabilities of the hardware, software and interface layers of a reality system to project a convincing stimuli onto the users. ${ }^{21}$ According to Brenda Laurel, a compelling VR experience has to follow certain engineering and conceptual principles that should facilitate to create a complete subjective and navigable environment reacting to user input and behavior. ${ }^{22}$ The immersion and presence are maintained not only by the representational function of the image but even more by the interactive potential of the real-time multi-sensory experience granted by the medium. This quality brings VR closer to an experience associated with interaction with a computer game or with a graphical interface, than with a passive spectatorship of non-interactive media like photography, film or animation. In VR the user, by using her own visual sensorium as a dynamic "camera" or framing apparatus, is both a director and a cinematographer. This feature of VR brings us closer to the situation that Oliver Grau named a defining point in the media history of the image- "dynamic virtual spaces". ${ }^{23} \mathrm{VR}$ environments are in fact zones of activity that simulate ontologies, create horizons of possibility - defined by affordances of computational system that can deliver specific visual, auditory and haptic cues to users. VR considered as a particular variation of software media experience makes use of advancements in software and hardware engineering that allow to create interactive, immersive computational environments that turn users into "immersants." ${ }^{4}$ The unique design affordances and constraints implemented in VR environments shape their status as cultural software that mediates people's interaction with media and other people. Therefore, if we consider VR environments as media interfaces, we are getting access to yet another perspective for analyzing different models of representing and accessing digital information in today's media ecology.

20 J.D. Bolter, R. Grusin, op. cit., p. 163; J. Jerald, op. cit., p. 45.

${ }^{21}$ J. Jerald, op. cit., pp. 45-46.

22 B. Laurel, “What Is Virtual Reality?”, Medium, https://medium.com/@blaurel/what-is-virtual-reality-77b876d829ba.

23 O. Grau, Virtual Art: From Illusion to Immersion, Cambridge, MA: MIT Press, 2003, p. 345.

${ }^{24}$ Ch. Davies, "Virtual Space", in: Space: In Science, Art and Society, ed. by R. Howell, G. Radick, F. Penz, Cambridge: Cambridge University Press, 2004, pp. 69-104. 


\section{VR as consumer-ready, immersive working environments?}

Conceptually, the idea to consider VR environments as media interface remediating spatial virtual environments seems valid, but if we look at today's consumer VR content landscape, it is still heavily oriented towards entertainment use cases. However, there's a particular category of software products that resonates with this idea, and at the same time, that is exposing the technological and conceptual limitations that will have to be overcome for that idea to materialize. These applications are often characterized as virtual desktop utility apps. Several such apps appeared on the market as a consequence of the launch of high-end PC-dependent VR headsets in early 2016. ${ }^{25}$ HTC Vive and Oculus Rift combined with content stores like Steam VR or Oculus Store laid the foundations for a consumer-friendly VR ecology of relatively standardized hardware and software solutions for accessing immersive media content. The emerging VR industry is trying to replicate the strategy that turned out to be successful on a mobile market, where thanks to standardized software ecosystems offered by Apple and Google, developers have been able to create millions of applications in the last decade. Oculus, HTC, Sony and other companies are encouraging developers to experiment and create different types of VR experiences targeted at their respective VR solutions which all offer fairly similar interaction models and visual fidelity. Utility apps are often regarded, even by their creators, as an experimental and niche category. In contrast to VR games or simulations which usually run in a full screen mode as another visual layer of an operating system and allow the user to be immersed in a narrative, game or in a multiplayer contest, utility applications are designed to be working environments. Apps like VR Toolbox: 360 Desktop, Multi VR.se or Virtual Desktop are regarded as Finders or Explorers of immersive environments. Their design rationale is to provide access to content stored on a PC (documents, media files) or accessed through PC (i.a. WWW) in a way that is suitable for a VR experience. As of 2018 they are the most sophisticated realization of an idea for a consumer-ready VR working environment or at least a spatial media viewer/ editor interface.

Despite minor differences, all analyzed apps share similarities in terms of design principles and implemented aesthetics / interaction models. The central representational element of their UI and its main metaphor is a 2D virtual window (or multiple windows) which can be scaled up and down and then dynamically positioned anywhere in a $3 \mathrm{D}$ environment. Similarly to desktop operating systems, the content displayed within their windows (frame) may change in real-time to display a web browser, a PC system's folder, or even a "full screen" content like game or video. The user can customize the virtual environment, which is in fact a spatial equivalent of a desktop wallpaper, a list of possible choices includes: an office, outer space, forest,

25 Software applications used as case study: DreamDesk VR, VR Toolbox: 360, MultiVR.se, Virtual Space, Bigscreen. 
cinema room etc. Adding or customizing 3D objects (simple meshes with textures) within the selected environment is also possible. A user can add an armchair, a desk or a lamp to make the space more personal. Due to hardware limitations (GPU processing power and display resolution) the image quality is by no means photorealistic. In immersive games or interactive experiences the image quality is not a crucial factor behind the feeling of presence as other cues (auditory, haptics, narrative etc.) make it more compelling. However, in the case of utility apps, factors such as low pixel density and resolution prevent the software from rendering text and image details with the fidelity known from traditional mobile or desktop devices. The condition of transparency that makes the desktop or mobile interface a working environment, is not well addressed in this case. ${ }^{26}$ However, this limitation can be overcome once the next generation of rendering software and displays with greater PPI (pixel per inch) ratio becomes available, for instance Vive Pro and Samsung Odyssey that debuted in 2018 address this issue. ${ }^{27}$

\section{VR working environments as a mediation layer for legacy, non-spatial media}

Conceptually, the utility apps could be characterized as yet another mediation layer between user and the environment of a PC operating system. Current iteration of this software is limited not only by technical affordances of the VR system (low resolution and pixel density, narrow field of view), but also by the "legacy" requirement it has to address. The fact that the main element of its interface is a virtual frame displaying content on a skeuomorphic representation, or a remediation of a flat screen, is hardly a coincidence or mere a designer's preference. In the field of software design skeuomorphs are often used as digital metaphors (elements of the graphical user interface) based on realistic representation techniques (of textures, fabrics, tools) in order to better visualize software functions and processes. They can be considered as material metaphors that "do not just signify and represent $[\ldots]$ but are also able to evoke acts in the material world with their mobilization of particular physical-material attributes" ${ }^{28}$, as they are more familiar to users than abstract elements. Consequently, skeuomorphs help users to navigate and use a computational device through its symbolic interface "which make(s) software accessible to users." ${ }^{29}$ Skeuomorphs are important components of the process of

26 J.D. Bolter, R. Grusin, op. cit., pp. 32, 23-24.

${ }_{27}$ See: https://www.vive.com/eu/product/vive-pro/ and https://www.samsung.com/us/computing/hmd/ windows-mixed-reality/xe800zaa-hclus-xe800zaa-hc1us/.

28 M. van den Boomen, Transcoding the Digital: How Metaphors Matter in New Media, Amsterdam: The Institute of Network Cultures, 2014, p. 55.

29 F. Cramer, M. Fuller, "Interface", in: Software Studies: A Lexicon, ed. by M. Fuller, Cambridge, MA: MIT Press, 2008, p. 149. 
remediation embedded into graphical user interfaces that represent different media and computational processes in a unified, windowed structure. ${ }^{30} \mathrm{~A}$ decade ago Apple applied skeuomorphism to iOS as one of the design principles. This approach helped familiarize users with a touch-based interface in mobile operating system. Today, analyzed utility apps like DreamDesk VR or VR Toolbox: 360 Desktop are following skeuomorphic aesthetics, replicating the elements of desktop and mobile GUI interfaces in a VR environment. Consequently, they effectively reduce the PC environment, with all its media content, to a single virtual frame (or a set of frames), which becomes a translation layer between a 3D (spatial) VR environment and a 2D (flat) computer environment. Apart from spherical video and CGI-based VR-ready content, all other types of digital media and information are designed to be viewed on a flat 2D screen, not a near-eye display device which is now a standard in consumer VR system. The representational model for today's web pages, images, icons is not native for an immersive environment. Consequently, the only way to display this type of content in a spatial virtual environment is to create a skeuomorphic representation of the screen - a virtual window. There is a long tradition that associates interfaces and interface elements with a "gateway"31 or "window", a point that grants users access to an environment beyond the surface of the screen. The screen itself, where "the contents move but the frame stays still" 32 , being a material form that embodies the interface, introduces an "ontological cut" 33 in a physical space in our offices, living rooms or movie theaters. That is the case not only of the last few decades with computer monitors in offices or television sets in living rooms. Oliver Grau traces the origins of the framed aesthetics in the $15^{\text {th }}$ century Renaissance paintings and frescos that depicted self-contained dimensional spaces thanks to perspective. ${ }^{34}$

The case study analysis has revealed that VR as a medium faces similar challenges as media before it. In its early days it has to accommodate the requirement of continuity — not only at a technical but particularly at the level of aesthetics and representation, just as film had to be compatible with still photography and digital video algorithms with film. At this point, VR is a hybrid medium spanned between the need for innovative representational and interaction paradigms and the requirement of maintaining support for digital/digitalized cultural content created in the past. This is clearly visible not only at the level of GUI that was analyzed above, but also at the level of physical interfaces which can be characterized as inconsistent at best. In order to navigate within the utility apps' virtual space a user is required to use a combination of physical controllers (custom hand-held gamepad, as well as PC mouse and keyboard) and skeuomorphic virtual keyboards and laser pointers displayed by

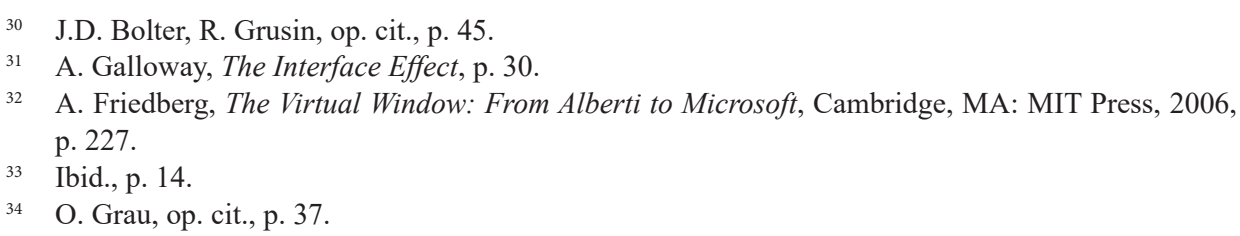


the HMD. As a result, there is little possibility to work with typical PC software that requires high precision or long-text input, the alternative is to constantly switch between virtual and physical controllers, which degrades the immersion and user experience as a whole. The next iterations of such applications should therefore put more attention into enabling kinaesthetic action considered as input method-deepen the degree of physical involvement while working in a virtual environment. ${ }^{35}$ However, the interaction models implemented in general utility applications will be always constrained by the affordances of hardware - accuracy of body (head, hands) tracking and precision of controllers.

\section{The "Holodeck" has not arrived yet}

At this point (late 2018) consumer-level VR solutions are not ready to offer a compelling working environment due to technological (visual fidelity, lack of high precision tracking and controllers) but also conceptual constraints (representational paradigm based on skeuomorphic representation of a pre-VR computer environment). At the same time the applications for VR as an entertainment platform appear to be more feasible. The study has revealed that it would be extremely challenging to convert virtual spaces into working environments without a compelling strategy for "translating" or "porting" pre-VR media content and software in a way so it can take advantage of the immersive space. This strategy may involve using voice interfaces, whole body tracking, procedural environment generation in real time. All these solutions are at least a few years away from realization. VR as a new type of immersive $3 \mathrm{D}$ medium that can be experienced in a volumetric physical space, requires a radical technological, conceptual and cultural shift in the paradigms for visual representation of information and ideas. This shift does not necessary involve modifying aesthetic practices that prevailed for centuries and have been grounded in perspective-based images displayed within a physical, yet flat framed surface. Therefore, future immersive environment can lay foundation to new models of data and content visualization and design. The popularity of creative applications like Tilt Brush (a room-scale 3D painting application by Google) or SketchUp VR (a tool for editing and visualizing architectural models from SketchUP in VR), already trigger the emergence of new art genres and design practices that make use of the spatial and real-time affordances of virtual reality. These affordances effectively make possible to manipulate the virtual environment, its objects and consider a whole virtual scene as an interface. Therefore, perhaps a more feasible solution would be not to remediate interfaces and visual metaphors designed for far eye displays and computational devices based on WIMP paradigms in a spatial environment, but to completely change the concept of a user interface. Experiments conducted by industry leaders and computer scientists proved that spatial virtual environments can actually become approximate and simplified

35 J. Woletz, "Interfaces of Immersive Media", Interface Critique Journal 2018, vol. 1, p. 108. 
simulations of actual physical environments. ${ }^{36}$ Such environments can be used to train autonomous agents and algorithms to better perform in physical environments at a later stage of their development. For instance, NVidia uses real-time CGI environments to train robots and autonomous vehicles as well as for experimental human-robot interaction. ${ }^{37}$

\section{Social and cultural impact of VR working environments}

While acknowledging the creative potential of virtual environments, we should not ignore some risks and challenges that go beyond the technological spectrum, due to the scope of the article these issues have not been fully articulated, however a few examples for further studies can be provided.

For instance, we could ask about the issues of data ownership and privacy of VR users. A proprietary software-based virtual environment can easily turn into the ultimate version of panopticon where not only the actions of users, but also their gaze and other biometric and behavioural data, can be turned into commodity. Another challenge would be to rethink the very practice of intellectual work with digital information-particularly within a team of workers (not necessarily human-only). Today VR is still in its infancy, however, even at this point one can predict that new affordances for data visualization, management and cooperation-based work in an immersive environment will demand for new workspace arrangements. Namely, the office of the future may have to resign from typical furniture and computer screens, in favour of truly open spaces that allow for mobility and adaptability. Physical location of working individuals may become even a lesser concern than it is now, new remote or home working job types may emerge. Clearly, we won't face this challenges in the very near future, however we should already start thinking about solutions. Consequently, we should not only focus on designing interfaces for virtual spatial environments using visual metaphors and established design / interaction models, but consider these spatial environments as ultimate interfaces and make use of the full spectrum of visual, auditory and haptic cues for machine-machine, human-machine and human-human interaction.

\section{Bibliography}

Andrade T., "Creating a Basic VR Demo Using Unreal”, Virtual Reality Pop 2016, https:// virtualrealitypop.com/start-a-vr-demo-using-the-unreal-engine-63d31 eeaf784.

36 J. Gregory, Game Engine Architecture, Boca Raton: Taylor \& Francis, CRC Press, 2018, p. 9.

37 K. Bye, "Training AI \& Robots in VR with Nvidia's project Holodeck", Voices of VR, episode \#623, 2018, https://bit.ly/2A7N44w and Nvidia Website, https://bit.ly/2pJv8r9. 
Akeley K., van Dam A., Foley J.D., Feiner S.K., Hughes J.F., McGuire M., Sklar D., Computer Graphics: Principles and Practice, Boston, MA: Addison-Wesley Longman Publishing Co., Inc., 2013.

Berry D.M., Critical Theory and the Digital, London: Bloomsbury, 2014.

Bolter J.D., Grusin R., Remediation: Understanding New Media, Cambridge, MA: MIT Press, 2003.

Boomen M. van den, Transcoding the Digital: How Metaphors Matter in New Media, Amsterdam: The Institute of Network Cultures, 2014.

Bye K., “Training AI \& Robots in VR with Nvidia's Project Holodeck", Voices of VR, episode \#623, 2018, https://bit.ly/2A7N44w.

Cramer F., Fuller M., "Interface”, in: Software Studies: A Lexicon, ed. by M. Fuller, Cambridge, MA: MIT Press, 2008.

Davies Ch., Osmose, http://www.immersence.com/osmose/.

Davies Ch., "Virtual Space", in: Space: In Science, Art and Society, ed. by R. Howell, G. Radick, F. Penz, Cambridge: Cambridge University Press, 2004, pp. 69-104.

Friedberg A., The Virtual Window: From Alberti to Microsoft, Cambridge, MA: MIT Press, 2006.

Fuller M., Media Ecologies: Materialist Energies in Art and Technoculture, Cambridge, MA: MIT Press, 2007.

Galloway A.R., Protocol, or, How Control Exists After Decentralization, Cambridge, MA: MIT Press, 2001.

Galloway A.R., The Interface Effect, Cambridge, UK: Polity, 2012.

Grau O., Virtual Art: From Illusion to Immersion, Cambridge, MA: MIT Press, 2003.

Gregory J., Game Engine Architecture, Boca Raton: Taylor \& Francis, CRC Press, 2018, p. 9. Heath A., "Mark Zuckerberg reveals that Facebook paid more than we thought for Oculus VR", Business Insider 2017, https://www.businessinsider.com/facebook-actually-paid-3-billion-for-oculus-vr-2017-1.

Israel S., Scoble R., The Fourth Transformation: How Augmented Reality \& Artificial Intelligence Will Change Everything, Austin: Patrick Brewster Press, 2016.

Jerald J., The VR Book: Human-Centered Design for Virtual Reality, New York: ACM Books, 2016.

Khronos Group, “OpenXR Overview”, https://www.khronos.org/openxr.

Laurel B., "What Is Virtual Reality?”, Medium, https://medium.com/@blaurel/what-is-virtual-reality-77b876d829ba.

Manovich L., The Language of New Media, Cambridge, MA: MIT Press, 2001.

Milgram P. et al., "Augmented Reality: A Class of Displays on the Reality-Virtuality Continuum", Proceedings of Telemanipulator and Telepresence Technologies 1994, pp. 23512334.

Rubin B.P., "Unity Dives into the Rift with Virtual Reality Tools for Games”, ReadWrite 2015, https://readwrite.com/2015/06/10/unity-adds-vr-support-to-game-engine/.

Whiting N., "Standardizing All the Realities: A Look at OpenXR", GDC 2018, https://www. youtube.com/watch? $\mathrm{v}=\mathrm{U}-\mathrm{CpA} 5 \mathrm{~d} 9 \mathrm{MjI}$.

Woletz J., "Interfaces of Immersive Media", Interface Critique Journal 2018, vol. 1, pp. 96110. 